\title{
PERTURBATIONS CAUSING OSCILLATIONS OF FUNCTIONAL-DIFFERENTIAL EQUATIONS
}

\author{
A. G. KARTSATOS AND M. N. MANOUGIAN
}

Abstract. Some new criteria are given for the oscillation of solutions of perturbed functional-differential equations of the form

$$
x^{(n)}+P(t) f(x(g(t)))=Q(t) .
$$

The results are new even in the case $g(t) \equiv t$, or when (I) is linear. The function $Q(t)$ does not have to be small or periodic.

1. Introduction. The first of the authors raised in [4] the following question: What kind of perturbations $Q(t)$ force all solutions of the equation

$$
x^{(n)}+P\left(t, x, x^{\prime}, \cdots, x^{(n-1)}\right)=Q(t), \quad n \geqq 2,
$$

to oscillate, although the homogeneous equation is not necessarily oscillatory? It is our intention here to answer this question for a large class of equations. Actually, we present our result in the case of a functional-differential equation of the type

$$
x^{(n)}+P(t) f(x(g(t)))=Q(t) .
$$

The theorems of this paper are entirely new even in the case $g(t) \equiv t$, or when $f$ is linear.

Possible generalizations and some examples are discussed at the end of the paper. For results related to the contents of this paper the reader is referred to Kartsatos [2], [3], [4], Teufel [6], Atkinson [1] for the case of an ordinary equation, and True [7], Kusano and Onose [5] for the case (I).

In what follows, use will be made of the following conditions:

(i) $P \in C[[0, \infty), R], R=(-\infty, \infty)$;

(ii) $g \in C[[0, \infty), R], \lim _{t \rightarrow \infty} g(t)=+\infty$;

(iii) $f \in C[\boldsymbol{R}, \boldsymbol{R}]$, increasing and $u f(u)>0$ for $u \in R$ with $u \neq 0$;

(iv) $Q \in C[[0, \infty), R]$.

Received by the editors May 16, 1973 and, in revised form, July 12, 1973.

AMS (MOS) subject classifications (1970). Primary 34C10.

Key words and phrases. Oscillation of solutions, nonoscillation of solutions, bounded solutions, nonlinear differential equations.

(c) American Mathematical Society 1974 
By a solution of (I) we mean a function $x \in C^{n}\left[\left[t_{x}, \infty\right), R\right]$, which satisfies (I) for all $t \in\left[t_{x}, \infty\right)$. Here $t_{x} \geqq 0$ depends on the particular solution $x(t)$. Let $\mathscr{F}$ denote the family of all such solutions of (I). A function $x \in \mathscr{F}$ is said to be "oscillatory" if it has an unbounded set of zeros in $\left[t_{x}, \infty\right)$. Equation (I) is called oscillatory if every $x \in \mathscr{F}$ is oscillatory.

2. Main results. The following theorem ensures the oscillation of all solutions of equation (I).

THEOREM 1. Along with the hypotheses (i)-(iv), assume that $P(t) \geqq 0$, $t \in[0, \infty)$ and that there exists a function $R \in C^{n}[0, \infty)$, oscillatory and, such that $R^{(n)}(t)=Q(t), t \in[0, \infty)$. Moreover, assume that for every $\lambda>0$,

$$
\begin{array}{r}
\limsup _{t \rightarrow \infty} \int_{0}^{t} P(s) f(\lambda+R(g(s))) d s=+\infty, \\
\liminf _{t \rightarrow \infty}^{t} P(s) f(-\lambda+R(g(s))) d s=-\infty .
\end{array}
$$

Then for $n$ even, equation (I) is oscillatory. For $n$ odd every $x \in \mathscr{F}$ is oscillatory, or such that $\lim [x(t)-R(t)]=0$ monotonically as $t \rightarrow \infty$. If the above integral conditions hold for $\lambda=0$, then (I) is oscillatory also for $n$ odd.

Proof. Assume that the above integral conditions hold for every $\lambda>0$ and that $n$ is even. Assume that (I) is not oscillatory. Let $x \in \mathscr{F}$ be such that $x(t)>0, t \geqq t_{0} \geqq t_{x}$. Let $u(t)=x(t)-R(t), t \in\left[t_{0}, \infty\right)$. Then $u(t)$ satisfies the equation

$$
u^{(n)}+P(t) f(u(g(t))+R(g(t)))=0 .
$$

Since $u(t)+R(t)>0$ for $t \in\left[t_{0}, \infty\right)$, it follows that there exists $t_{1} \geqq t_{0}$ such that $u(g(t))+R(g(t))>0$ for $t \geqq t_{1}$. Thus,

$$
u^{(n)}(t)=-P(t) f(u(g(t))+R(g(t))) \leqq 0, \quad t \geqq t_{1} .
$$

Consequently, all the derivatives of $u(t)$ up to the order $n$ are eventually of one sign and none of them is identically zero on an infinite interval, because this would imply the same for the derivative of order $n$, a contradiction to the first of the integral assumptions. Furthermore, $u(t)$ is strictly increasing, or strictly decreasing for all large $t$. Assume for the moment that $u(t)<0$ for all large $t$. Then there exists $t_{2} \geqq t_{1}$ such that $u(g(t))<0$ for $t \geqq t_{2}$. Thus, $0>u(g(t))>-R(g(t))$, a contradiction to the oscillatory character of $R(t)$. It follows that $u(t)>0$ for all large $t$, and this implies (cf, also Kartsatos [3, Theorem 2]) that $u^{\prime}(t)>0$ and $u^{(n-1)}(t)>0$ for all large $t$, Thus, there exists $t_{2} \geqq t_{1}$ such that

$$
u^{(n-1)}(t)>0 \text { and } u(g(t)) \geqq \lambda>0 \text { for } t \geqq t_{2},
$$


where $\lambda$ is a constant. Integrating (1) we obtain, for $t \geqq t_{2}$,

$$
\begin{aligned}
u^{(n-1)}(t) & =u^{(n-1)}\left(t_{2}\right)-\int_{t_{2}}^{t} P(s) f(u(g(s))+R(g(s))) d s \\
& \leqq u^{(n-1)}\left(t_{2}\right)-\int_{t_{2}}^{t} P(s) f(\lambda+R(g(s))) d s .
\end{aligned}
$$

This easily implies $\lim \inf _{t \rightarrow \infty} u^{(n-1)}(t)=-\infty$, a contradiction to the first of (3). Consequently, $u(t)$ cannot be positive, or negative, or oscillatory for all large $t$, i.e., $u(t)$ does not exist under the assumption $x(t)>0$, $t \geqq t_{0}$. A similar situation appears in the case $x(t)<0, t \geqq t_{0}$, and this completes the proof of the theorem in the case of even $n$. Exactly the same argument applies in the case of odd $n$, except the fact that $u^{\prime}(t)$ could be eventually negative when $u(t)>0$ for all large $t$, or eventually positive when $u(t)<0$ for all large $t$. Both cases imply $\lim u(t)=\lim [x(t)-R(t)]=0$ monotonically as $t \rightarrow \infty$. For such considerations the reader is referred to the proof of Theorem 1 in Kartsatos [4]. Now if the integral conditions hold for $\lambda=0$, then they hold for all $\lambda>0$, and the theorem is true for the case of even $n$. However, if $n$ is odd we obtain from the equation in (4)

$$
u^{(n-1)}(t) \leqq u^{(n-1)}\left(t_{2}\right)-\int_{t_{2}}^{t} P(s) f(R(g(s))) d s,
$$

for all $t \geqq$ (some) $t_{2}$. This implies $\lim _{t \rightarrow \infty} u^{(n-1)}(t)=-\infty$, or $\lim _{t \rightarrow \infty} u(t)=$ $-\infty$, a contradiction to the positivity of $u(t)$. An analogous situation holds in the case of an eventually negative $u(t)$. Consequently, $x(t)$ is oscillatory, and the proof is complete.

We now give two useful corollaries to the above theorem.

COROLlaRY 1. Assume that $f(u)=u^{2 q+1}$, where $q$ is a nonnegative integer. Assume further that the hypotheses (i)-(iv) are satisfied, $P(t) \geqq 0$ for $t \geqq 0$, and $Q, R$ are as in Theorem 1. Moreover, let

$$
\int_{0}^{\infty} P(t) d t=+\infty, \quad \int_{0}^{\infty} P(t)|R(g(t))|^{m} d t<\infty,
$$

for every $m=1,2, \cdots, 2 q+1$. Then the conclusion of Theorem 1 concerning the case $\lambda \neq 0$ is satisfied.

Proof. It suffices to observe that, for a real $\lambda \neq 0$,

$$
(\lambda+R(g(t)))^{2 q+1}=\sum_{k=0}^{2 q+1}\left(\begin{array}{c}
2 q+1 \\
k
\end{array}\right) \lambda^{2 q+1-k}[R(g(t))]^{k} .
$$


COROLlaRY 2. Let the assumptions of Corollary 1 be satisfied except (6). Let

$$
\begin{gathered}
\underset{t \rightarrow \infty}{\limsup } \int_{0}^{\infty} P(t)[R(g(t))]^{m} d t=+\infty, \\
\underset{t \rightarrow \infty}{\liminf } \int_{0}^{\infty} P(t)[R(g(t))]^{m} d t=-\infty
\end{gathered}
$$

for some even integer $m$ with $2 \leqq m \leqq 2 q$, and

$$
\int_{0}^{\infty} P(t)|R(g(t))|^{j} d t<+\infty
$$

for each $j=0,1,2, \cdots, m-1, m+1, \cdots, 2 q+1$.

Then the conclusion of Theorem 1 concerning the case $\lambda \neq 0$ is satisfied.

Proof. Another application of formula (7), since every even power of $R(g(t))$ is multiplied by an odd power of $\lambda$.

The effect of "weighted" integral conditions on the bounded solutions of (I) is given by the following

THEOREM 2. Let the assumptions of Theorem 1 hold with $R$ bounded and the integral conditions replaced by

$$
\begin{gathered}
\limsup _{t \rightarrow \infty} \int_{0}^{t} s^{m_{1}} P(s) f(\lambda+R(g(s))) d s=+\infty, \\
\liminf _{t \rightarrow \infty}^{t} \int_{0}^{t} s^{m_{2}} P(s) f(-\lambda+R(g(s))) d s=-\infty,
\end{gathered}
$$

where $m_{1}, m_{2}$ are integers with $1 \leqq m_{1}, m_{2} \leqq n-1$.

Then (a) if (8), (9) hold for every $\lambda>0$, every bounded $x \in \mathscr{F}$ is oscillatory for $n$ even, and every bounded $x \in \mathscr{F}$ is oscillatory, or such that $\lim [x(t)-R(t)]=0$ monotonically as $t \rightarrow \infty$ for $n$ odd; (b) if (8), (9) hold for $\lambda=0$, every bounded $x \in \mathscr{F}$ is oscillatory.

Proof. Assume that $n$ is even and that $x(t), u(t)$ are as in the proof of Theorem 1 up to the inequalities (3). Then consider the function $t^{m_{1}} u^{(n-1)}(t)$. Differentiation of this function gives

$$
\left[t^{m_{1}} u^{(n-1)}(t)\right]^{\prime}=-t^{m_{1}} P(t) f(u(g(t))+R(g(t)))+m_{1} t^{m_{1}-1} u^{(n-1)}(t),
$$

and integration from $t_{2}$ to $t \geqq t_{2}$ gives

$$
\begin{aligned}
t^{m_{1}} u^{(n-1)}(t)= & t_{2}^{m_{1}} u^{(n-1)}\left(t_{2}\right)-\int_{t_{2}}^{t} s^{m_{1}} P(s) f(u(g(s))+R(g(s))) d s \\
& +m_{1} \int_{t_{2}}^{t} s^{m_{1}-1} u^{(n-1)}(s) d s .
\end{aligned}
$$


This implies (by condition (8))

$$
\liminf _{t \rightarrow \infty}\left[t^{m_{1}} u^{(n-1)}(t)-m_{1} \int_{t_{2}}^{t} s^{m_{1}-1} u^{(n-1)}(s) d s\right]=-\infty .
$$

However, since $u^{(n-1)}(t)>0$ for $t \geqq t_{2}$, and the integral in (11) is an increasing function of $t$, we have

$$
\lim _{t \rightarrow \infty} \int_{t_{2}}^{t} s^{m_{1}-1} u^{(n-1)}(s) d s=+\infty .
$$

The proof now continues as in Theorem 1 of Kartsatos [3]. If $x(t)$ is bounded then $u(t)$ is also bounded and $(-1)^{k} u^{(k)}(t)<0$ for $k=1,2, \cdots$, $n-1$ and $t \in\left[t_{2}, \infty\right)$, and successive integration by parts of the integrand in (12) yields eventually

$$
\begin{aligned}
\lim _{t \rightarrow \infty} \int_{t_{2}}^{t} u^{(n-m)}(s) d s & =+\infty, \quad \text { if } m \text { is odd, } \\
& =-\infty, \quad \text { if } m \text { is even, }
\end{aligned}
$$

which is a contradiction to the boundedness of $u(t)$ :

$$
\lim _{t \rightarrow \infty}\left[u^{(n-m-1)}(t)-u^{(n-m-1)}\left(t_{2}\right)\right]= \pm \infty .
$$

In order to avoid repetition, we omit the rest of the proof concerned with the case of eventually negative $u(t)$, or $n$ odd, or $\lambda=0$.

It is natural to expect now that similar results hold in the case of a nonpositive $P(t)$. As the following example indicates, this is not always the case.

EXAmple 1. Consider the second order equation

$$
x^{\prime \prime}-\left(e^{t}-Q(t)\right) e^{-t} x=Q(t),
$$

with $R(t)=e^{t / 2} \sin t$ and $Q(t)=R^{\prime \prime}(t)$. Then it is easy to check that the integral conditions of Theorem 1 hold for $\lambda=0$. However, (13) has the nonoscillatory solution $x(t)=e^{t}$.

Nevertheless, there are some cases of importance where Theorems 1 and 2 have analogues. The following theorem ensures the oscillation of all bounded solutions of the equation

$$
x^{(n)}-P(t) f(g(t))=Q(t)
$$

with $P(t) \geqq 0$.

THEOREM 3. Theorem 2 holds for the equation (II), if $m_{1}, m_{2}$ in (8), (9) are allowed to be zero, and if "odd" is replaced everywhere by "even" and conversely. 
Proof. Assume that $x(t), t \in\left[t_{x}, \infty\right)$, is a bounded nonoscillatory solution of (II). Then, without loss of generality, we assume that $x(t)>0$, $t \in\left[t_{1}, \infty\right), t_{1} \geqq t_{x}$. Then $u(t)=x(t)-R(t)$ is a bounded solution of the equation

$$
u^{(n)}-P(t) f(u(g(t))+R(g(t)))=0,
$$

for which $u(g(t))+R(g(t))>0$ for every $t \geqq t_{2} \geqq t_{1}$. Consequently, $u^{(n)}(t) \geqq 0$ for every $t \geqq t_{2}$, which implies that all the derivatives $u^{(i)}(t), i=0,1, \cdots, n$, are of fixed sign for all large $t$, and no two consecutive derivatives are of the same sign for all large $t$, for this would force $u(t)$ to diverge to $\pm \infty$ as $t \rightarrow \infty$. Thus, $u^{(n-1)}(t) \leqq 0$ for $t \geqq t_{3} \geqq t_{2}$. Now assume that the integral condition (8) holds for every $\lambda>0$. Then, as in the proof of Theorem 2 , we obtain

$$
\begin{aligned}
t^{m_{1}} u^{(n-1)}(t)= & t_{3}^{m_{1}} u^{(n-1)}\left(t_{3}\right)+\int_{t_{3}}^{t} s^{m_{1}} P(s) f(u(g(s))+R(g(s))) d s \\
& +m_{1} \int_{t_{3}}^{t} s^{m_{1}-1} u^{(n-1)}(s) d s, \quad t \geqq t_{3} .
\end{aligned}
$$

Now assume that $n=$ odd. Then since $u^{\prime}(t) \geqq 0$ for $t \geqq t_{3}, u(g(t)) \geqq \lambda>0$ for $t \in\left[t_{3}, \infty\right)\left(t_{3}\right.$ can be chosen a priori this way). Thus, from (15) we obtain

$$
\begin{aligned}
t^{m_{1}} u^{(n-1)}(t)-m_{1} & \int_{t_{3}}^{t} s^{m_{1}-1} u^{(n-1)}(s) d s \\
& \geqq t_{3}^{m_{1}} u^{(n-1)}\left(t_{3}\right)+\int_{t_{3}}^{t} s^{m_{1}} P(s) f(\lambda+R(g(s))) d s
\end{aligned}
$$

for $t \geqq t_{3}$, which implies $\lim _{t \rightarrow \infty} \int_{t_{3}}^{t} s^{m_{1}-1} u^{(n-1)}(s) d s=-\infty$, a contradiction as in Theorem 2. We omit the rest of the proof.

3. Discussion-Examples. It is possible to extend the present results to equations of the form

$$
x^{(n)}+P_{0}(t, \tilde{x}(t), \tilde{x}(g(t)))=Q(t)
$$

where $\tilde{x}(t)=\left(x(t), x^{\prime}(t), \cdots, x^{(n-1)}(t)\right)$, under suitable assumptions on the function $P_{0}$. For example, $P_{0}$ could be considered as bounded above and below by functions of the form $P(t) f(x(g(t)))$. A lot of open problems arise now with respect to the choice of the perturbation $Q(t)$. For example, there exist oscillating perturbations, which cannot be represented as $n$th derivatives of oscillating functions. For example, the function $Q(t) \equiv$ $\frac{1}{2}+2 \sin t$. Any function $R(t)$ with $R^{(n)}(t)=Q(t)$, would have to satisfy $R^{(n-1)}(t)=\frac{1}{2} t-2 \cos t+C\left(C\right.$ constant) for all large $t$, i.e., $\lim _{t \rightarrow \infty} R(t)=\infty$. However, the equation

$$
x^{\prime \prime}-x=\frac{1}{2}+2 \sin t
$$


has all its bounded solutions oscillatory. In fact, the general solution of (16) is $x(t)=c_{1} e^{t}+c_{2} e^{-t}-\sin t-\frac{1}{2}$. Consequently, there are large classes of equations to be studied under the above considerations. In short, it should be possible to study the oscillatory character of (II) without using the transformation $u(t)=x(t)-R(t)$. Another open problem is the following: Assuming that the homogeneous equation is oscillatory, what kind of perturbations $Q(t)$ stop the oscillation of all, or part of the solutions of (II)?

In view of Corollaries 1 and 2 it is easy to give examples of equations satisfying all the assumptions of Theorem 1 . An equation satisfying all the assumptions of Theorem 2 is the following

$$
x^{\prime \prime}+\left[\frac{1}{4}\left(1+t^{2}\right)\right] x=\left[t^{-\alpha} \sin t\right]^{\prime \prime}, \quad t \geqq 1,
$$

where $\alpha$ is a constant with $0<\alpha<1$. It follows that every solution of (17) is either unbounded or bounded and oscillatory.

The authors wish to express their thanks to the referee for his helpful suggestions concerning the style of the paper.

\section{REFERENCES}

1. F. V. Atkinson, On second-order differential inequalities (to appear).

2. A. G. Kartsatos, A contribution to the investigation of the oscillations and asymptotic behavior of the solutions of ordinary differential equations, Bull. Soc. Math. Grèce 10 (1969), fasc. 2, 1-50, 135. (Greek) MR 43 \#618.

3. - On the maintenance of oscillations of nth order equations under the effect of a small forcing term, J. Differential Equations 10 (1971), 355-363. MR 44 \#5556.

4. - Maintenance of oscillations under the effect of a periodic forcing term, Proc. Amer. Math. Soc. 33 (1972), 377-383.

5. T. Kusano and $\mathrm{H}$. Onose, Oscillations of functional differential equations with retarded arguments, J. Differential Equations (to appear).

6. H. Teufel Jr., Forced second order nonlinear oscillation, J. Math. Anal. Appl. 40 (1972), 148-152.

7. E. True, Doctoral Dissertation, Montana State University, Bozeman, Mt., 1972.

Department of Mathematics, University of South Florida, Tampa, Florida 33620 